\title{
Efecto del encalamiento de suelos sulfatados ácidos en Córdoba (Colombia) sobre el fruto de piña (Ananas comosus (L.) Merr.)
}

\section{Effect of liming of acid sulfate soils in Cordoba (Colombia) on pineapple (Ananas comosus (L.) Merr.) fruit}
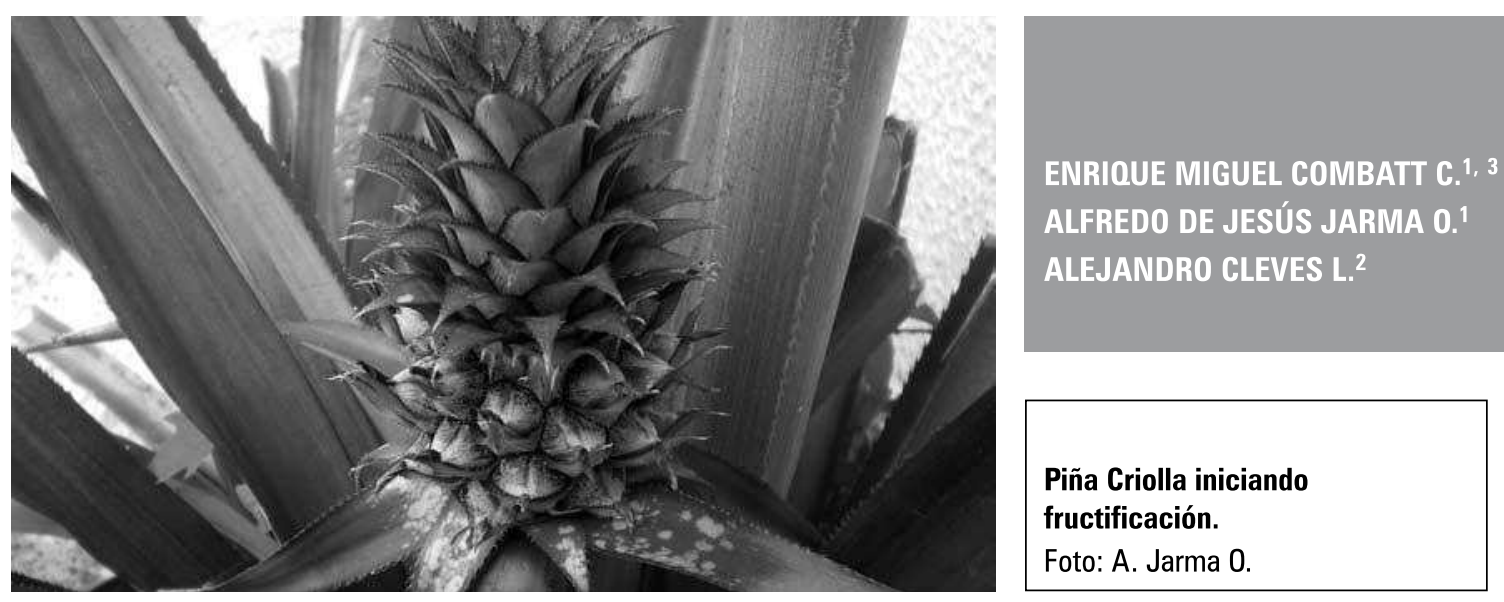

\section{RESUMEN}

En el departamento de Córdoba (Colombia) existen entre 20.000 y 30.000 ha de suelos sulfatados ácidos (SSA), los cuales causan toxicidad principalmente por la elevada solubilidad del aluminio, poca disponibilidad de fósforo, baja saturación de las bases y salinidad, entre otros. Lo anterior es más grave aún si se considera que existe un gran vacío en el conocimiento del comportamiento de especies como la piña en este tipo de suelos. Por lo anterior, el presente trabajo determinó el efecto de tres niveles de encalamiento en SSA del municipio de Ciénaga de Oro (Córdoba), sobre algunos aspectos de la calidad del fruto de dos variedades de piña Cayena lisa y Reina de Zarandelo o Criolla. Se realizó un diseño de parcelas divididas bajo bloques completamente al azar en donde las parcelas principales fueron los genotipos y las subparcelas las diferentes dosis de cal agrícola $\left(3,6\right.$ ó $9 \mathrm{t} \mathrm{ha}^{-1} \mathrm{de}_{\mathrm{CaCO}_{3}}$ ). Los resultados indicaron que tanto el diámetro ecuatorial como la firmeza del fruto fueron dependientes solamente de la variedad; el diámetro del fruto de Cayena lisa fue superior en $10 \%$ y la firmeza de 'Criolla' fue superior en $26 \%$. Los valores de $\mathrm{pH}$ del fruto indicaron que esta variable es afectada por la interacción del genotipo con la dosis de encalamiento, registrando un comportamiento menos estable la variedad 'Cayena lisa' cuando se varían los niveles de cal en el suelo.

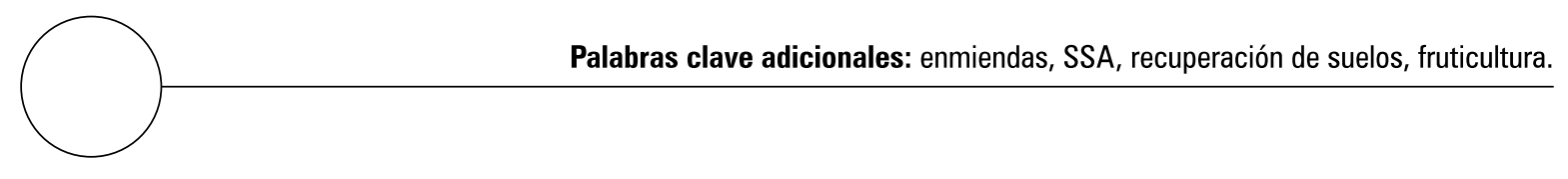

Facultad de Ciencias Agrícolas, Universidad de Córdoba, Montería (Colombia).

Facultad Seccional Duitama, Universidad Pedagógica y Tecnológica de Colombia, Duitama (Colombia).

Autor para correspondencia. ecombatt@sinu.unicordoba.edu.co 


\section{ABSTRACT}

In the province of Cordoba (Colombia), exist between 20,000 and 30,000 ha of acid sulphate soils (ASS). The chemical problems that present ASS for crop production include toxicity, principally due to the high solubility of aluminum, low availability of phosphorus, low saturation of bases, salinization, among others. There is a lack of knowledge about the behavior of species, such as pineapple in this type of soils, for which was studied the effect of three liming levels of ASS located in the municipality Cienaga de Oro on yield and quality of fruits of two pineapple varieties Cayena Lisa and Reyna de Zarandelo or Criolla. The experiment was done in a completely randomized block design, where the principal plots were genotypes and the subplots were the different doses of agricultural lime $\left(3,6\right.$ or $\left.9 \mathrm{t} \mathrm{ha}^{-1} \mathrm{CaCO}_{3}\right)$. The results indicated that both the equatorial diameter and the fruit firmness were dependent only on variety; the fruit diameter of 'Cayena Lisa' was $10 \%$ wider than that of fruits of 'Criolla' and firmness values of 'Criolla' was $26 \%$ higher than that values of Cayena. The $\mathrm{pH}$ values indicated that this variable is affected by the interaction genotype-dose of liming registering a less stable behavior of the variety Cayena Lisa when the levels of lime are changed in soil.

Additional key words: amendments, ASS, soils recovery, pomology.

Fecha de recepción: 03-09-2008

Aprobado para publicación: 02-12-2008

INTRODUCCIÓN

La toxicidad por aluminio limita en $30 \%$ la producción de los cultivos en los suelos arables del mundo que incluyen muchas áreas del trópico y subtrópico, donde predomina el aluminio en forma de $\mathrm{Al}^{3+}$, lo cual afecta el crecimiento de las raíces y aumenta la deficiencia de nutrientes (Nowak y Friend, 2005; Pérez y Martínez, 2006).

Los suelos sulfatados ácidos (SSA) reducen considerablemente la productividad agrícola en el trópico; el ácido soluble disminuye el $\mathrm{pH}$ a tal punto que las altas concentraciones de iones de hidrógeno en la solución del suelo promueven el ataque a la estructura cristalina, lo que permite que se liberen iones de aluminio que luego pasan a ocupar lugares en los sitios de cambio (Bello y Gómez, 2001). Los procesos de oxidación tam- bién contribuyen en estos suelos a la composición de sales ácidas influyendo en conductividades eléctricas hasta de $10 \mathrm{dS} \mathrm{m} \mathrm{m}^{-1}$, además de afectar su estructura, constituyendo suelos masivos e impermeables por efectos cementantes y floculantes de los compuestos de oxihidróxido de hierro y aluminio (NSWDLWC, 1999).

Algunas especies han creado mecanismos naturales de adaptación a este tipo de suelos. Diversos autores indican procesos eficientes como la exudación del aluminio a través de ácidos orgánicos por las raíces, aunque esto no está completamente identificado (Watanabe y Osaki, 2002; Taylor et al., 2000). Otros mecanismos de resistencia al aluminio también han sido reportados, como el incremento del $\mathrm{pH}$ en la rizosfera y la 
formación de complejos de aluminio con ácidos orgánicos o quelatos, que secuestran esos complejos en las vacuolas.

Para contrarrestar los efectos de los SSA, generalmente se ha usado el encalamiento con el propósito de neutralizar la acidez ocasionada por el hidrógeno, aluminio, manganeso, hierro y ácidos de la materia orgánica, con el fin de permitir el crecimiento normal de las plantas (Welch et al., 2009). Sin embargo, algunos autores dicen que esta práctica es a menudo cara y poco eficiente en muchas partes de los trópicos (Sierra et al., 2003), y otros recomiendan realizar lavados antes de encalar y posteriormente fertilizar (Mathew et al., 2001).

En Colombia, la piña (Ananas comosus (L.) Merr.) se cultiva en una amplia gama de suelos, sin que en muchos casos se hagan las aplicaciones o correctivos necesarios, y peor aún, no existe información sobre el comportamiento de esta en SSA. Se usan suelos con pH desde 3,7 en Lebrija (Santander), hasta 7,5, en Yotoco (Valle del Cauca) y contenidos nutricionales muy diferentes (Salazar, 1994). La piña es una planta exigente en nutrientes; extrae aproximadamente 350, 30 y 500 $\mathrm{kg} \mathrm{ha}^{-1}$ de $\mathrm{N}, \mathrm{P}$ y $\mathrm{K}$ respectivamente en cultivos con una densidad de población de 50.000 plantas/ ha (Teixeira et al., 2002). El peso y la calidad del fruto de la piña dependen, entre otros factores, especialmente del estado nutricional y el estado de crecimiento alcanzado por la planta cuando se da la diferenciación floral (Cunha y Cabral, 1999). Según Paula et al. (1998), los nutrientes más exigidos por piña son $\mathrm{K}, \mathrm{N}$ y Ca.

El fruto de la piña es una infrutescencia relativamente comprimida formada por "frutillos" que maduran en orden creciente de abajo hacia arriba (Coppens y Leal, 2003), de modo que los de la parte inferior tienen edades fisiológicas mayores que los de las partes medianas y superior, lo que puede resultar en variaciones muy significativas en atributos de calidad de la pulpa del fruto (Reinhardt, 2004).

\section{MATERIALES Y MÉTODOS}

El trabajo se realizó en un SSA del municipio de San Carlos, Vereda La Coroza (departamento de Córdoba, Colombia), ubicada a 0855'47,5” N y $075^{\circ} 42^{\prime} 16,5^{\prime \prime} \mathrm{W}$ a una altura de $15 \mathrm{msnm}$, humedad relativa promedio de $85 \%$, precipitación de $1.250 \mathrm{~mm}$ año-1 y temperatura media anual de $28^{\circ} \mathrm{C}$. Se usó un diseño experimental de bloques completos al azar con estructura de parcelas divididas, donde las parcelas principales fueron los genotipos de piña 'Cayena lisa' y 'Reina de Zarandelo' o Criolla y las subparcelas fueron las dosis de cal (3, 6 y $\left.9 \mathrm{t} \mathrm{ha}^{-1}\right)$. La siembra se realizó $30 \mathrm{~d}$ después del encalamiento (dde). La fertilización se realizó con base en los resultados del análisis de suelo en cantidades por hectárea de $69 \mathrm{~kg}$ de $\mathrm{N}$ (urea), $46 \mathrm{~kg}$ de fosfato diamónico en presiembra $\left(\mathrm{P}_{2} \mathrm{O}_{5}\right)$ y $60 \mathrm{~kg}$ de $\mathrm{K}$ como $\mathrm{K}_{2} \mathrm{O}$.

Al observar un término medio de maduración de los frutos (alrededor de $150 \mathrm{~d}$ después de la formación de la inflorescencia), sobre cinco frutos escogidos al azar por tratamiento se midieron las variables resistencia a la penetración, medida en $\mathrm{kg} \mathrm{cm}^{-2}$ y diámetro ecuatorial, medido en $\mathrm{cm}$ y pH. Para el análisis estadístico se usó el paquete Statistical Analysis System (SAS), versión 6.0 de la Universidad de Córdoba; la comparación de los efectos simples se realizó mediante la prueba de comparación de medias DMS a un nivel del $5 \%$ de probabilidad y las interacciones que resultaron se descompusieron al mismo nivel de significancia con tablas de doble entrada, midiendo los efectos de los niveles del factor genotipo dentro de los niveles del factor dosis y viceversa.

\section{RESULTADOS Y DISCUSION}

El análisis de los cuadrados medios de las variables dependientes indicó que la resistencia y el diámetro ecuatorial del fruto fueron afectados significativamente de manera exclusiva por el genotipo $(P \leq 0,05)$, pero el $\mathrm{pH}$ registró un com- 
portamiento que es afectado por la interacción del genotipo y la dosis de cal utilizada (tabla 1).

\section{Resistencia y diámetro ecuatorial del fruto}

Estas variables solo fueron afectadas por el efecto del genotipo indicando que no son influenciadas por el nivel de encalamiento y que sus valores son explicados por el grado de madurez del fruto de cada variedad, por lo que solo se discutirá este aspecto. Los resultados indicaron que la variedad Criolla registró mayores promedios de resistencia a la penetración $(P \leq 0,05)$ respecto a Cayena lisa $\left(8,47\right.$ y $6,29 \mathrm{~kg} \mathrm{~cm}^{-2}$ respectivamente) (tabla 2). También se observan los valores de diámetro ecuatorial del fruto, registrándose un comportamiento similar y consecuente con el de resistencia, en el sentido que los frutos con menor grado de madurez en este caso de la variedad criolla, registraron $0,9 \mathrm{~cm}$ menos de longitud $(P \leq 0,05)$, respecto a la variedad Cayena lisa que mostró valores promedio de $9,19 \mathrm{~cm}$. Esta última variedad por tanto, registraría frutos de piña de mayor diámetro ecuatorial y con un mayor grado de madurez respecto a los de criolla en esta región del país, cuando es cultivada en SSA de manera independiente al nivel de encalamiento.

Tabla 1. Cuadrados medios y significancia estadística del diámetro ecuatorial, resistencia a la penetración y pH del fruto de dos variedades de piña cultivadas en suelos sulfatados ácidos de Ciénaga de Oro (Córdoba, Colombia).

\begin{tabular}{|l|c|r|c|}
\hline \multicolumn{1}{|c|}{ F de V } & Resistencia & $\begin{array}{r}\text { Diámetro } \\
\text { ecuatorial }\end{array}$ & \multicolumn{1}{c|}{ pH } \\
\hline Variedad (V) & $38,06^{*}$ & $13,08^{*}$ & $0,2363^{* *}$ \\
\hline Dosis (D) & $4,68 \mathrm{~ns}$ & $2,97 \mathrm{~ns}$ & $0,0104^{* *}$ \\
\hline VxD & $0,91 \mathrm{~ns}$ & $1,51 \mathrm{~ns}$ & $0,0136^{* *}$ \\
\hline CV (\%) & 13,1 & 11,7 & 11,4 \\
\hline
\end{tabular}

ns $=$ no significativo; ${ }^{*} y{ }^{*}$ significativos al 10 y $5 \%$ respectivamente según el test de DMS.

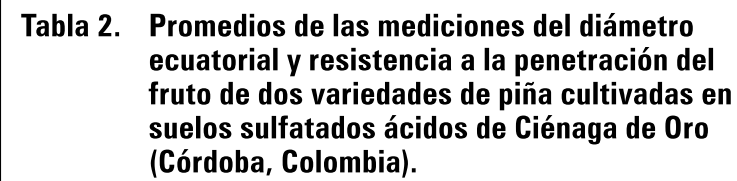

\begin{tabular}{|l|l|l|}
\hline Cayena lisa & $\begin{array}{c}\text { Resistencia } \\
\left(\mathrm{kg} \mathrm{cm}^{-2}\right)\end{array}$ & $\begin{array}{c}\text { Diámetro ecuatorial } \\
(\mathrm{cm})\end{array}$ \\
\hline Criolla & $8,29 \mathrm{a}( \pm 0,727)$ & $9,19 \mathrm{a}( \pm 0,769)$ \\
\hline CV $(\%)$ & 23 & $8,31 \mathrm{~b}( \pm 1,361)$ \\
\hline
\end{tabular}

*Promedios con letras distintas en la misma columna indican diferencia significativa según el test protegido de DMS $(P \leq 0,05)$.

A pesar de que algunos estudios confirman que a los 150 d después de la aparición de la inflorescencia se presenta un estado de madurez intermedio en términos generales para la especie (Arias y Toledo, 2000), estos resultados serían los primeros reportes sobre la fenología y la resistencia del fruto de estas variedades para las condiciones de Córdoba, por lo que es difícil referenciarlos con otros trabajos.

Aunque el estado de madurez en piña depende en gran medida del genotipo (Carvalho et al., 2005), es importante considerar que las condiciones ambientales y algunos factores de estrés como la salinidad del suelo, pueden inducir mayor síntesis de etileno endógeno que probablemente derivan en una mayor precocidad a floración (Bernal, 2006; Bartholomew y Malézieux, 1994; Bartholomew y Kadzimin, 1997).

Trabajos que describan el desarrollo del fruto y de sus características en esta región del país son escasos. Algunos valores de resistencia en piña (Ananas comosus cv. MD-2) han sido reportados por De la Cruz et al. (2007), en trabajos de poscosecha con rangos que van desde 12 (protegidas del daño por frío) a $2 \mathrm{~kg} \mathrm{~cm}^{-2}$ (sin proteger). Por su parte, en un trabajo publicado por la Universidad de Antioquia (1993), se reportan valores de $10,43 \mathrm{~cm}$ en el diámetro central de la variedad Cayena lisa para las condiciones del Valle del Cauca. 


\section{$\mathrm{pH}$ del fruto}

Tal como se indicó anteriormente, los resultados del $\mathrm{pH}$ del fruto fueron diferenciales con la variedad al interactuar con el nivel de encalamiento. La tabla 3 registra la descomposición de la interacción, la cual se analizó inicialmente en función de la respuesta de la variedad dentro de cada nivel de encalamiento (tabla $3 a$ ) y posteriormente en función de la respuesta de cada nivel de encalamiento dentro del factor variedad (tabla 3b).

Al considerar el efecto de la variedad dentro de cada dosis de encalamiento, los resultados indicaron que las variedades se comportaron de manera similar en cualquier nivel de encalamiento siendo siempre el valor del $\mathrm{pH}$ del fruto menor en 'Criolla' respecto a 'Cayena lisa'. Estos resultados no explicarían los efectos significativos registrados en el análisis de la interacción, por lo que se estudiaron los efectos de las dosis dentro de las variedades (tabla $3 b)$. En este caso, sí se detectaron diferencias $(P \leq 0,05)$, que indican que para los frutos de la variedad Cayena lisa el $\mathrm{pH}$ presenta un comportamiento errático en función del nivel de cal aunque con tendencia a ser menor en la medida que se aumenta el encalamiento. A diferencia de Cayena lisa, para la variedad Criolla, el $\mathrm{pH}$ es significativamente mayor cuando se aplica cal en cualquiera de las tres dosis estudiadas, sugiriendo una respuesta directa del $\mathrm{pH}$ del fruto al encalamiento.

Lo anterior indicaría que la eficiencia de la práctica de encalamiento podría ser explicada en parte porque la cal influyó en la neutralización de la acidez generada por el $\mathrm{Al}^{3+}$ permitiendo la disponibilidad de otros nutrientes como el nitrógeno, lo cual tendría consecuencias directas sobre el pH del fruto (Watanabe y Osaki, 2002; Taylor et al., 2000). Este efecto podría estar explicado además porque la variedad Criolla, por presentar mayores niveles de adaptación a los suelos y condiciones
Tabla 3. Análisis de la interacción variedad $\mathrm{x}$ dosis del pH del fruto de dos variedades de piña cultivadas en suelos sulfatados ácidos de Ciénaga de Oro (Córdoba-Colombia).

\section{a. Análisis del efecto de la variedad dentro del nivel de encalamiento}

\begin{tabular}{|l|c|c|c|c|}
\hline \multirow{2}{*}{ Variedad } & \multicolumn{4}{|c|}{ Dosis de cal $\left(\mathrm{t} \mathrm{ha}^{-1}\right)$} \\
\cline { 2 - 5 } & 0 & 3 & 6 & 9 \\
\hline Cayena lisa & $4,12 \mathrm{a}$ & $4,12 \mathrm{a}$ & $4,08 \mathrm{a}$ & $4,06 \mathrm{a}$ \\
\hline Criolla & $3,82 \mathrm{~b}$ & $3,97 \mathrm{~b}$ & $3,97 \mathrm{~b}$ & $3,92 \mathrm{~b}$ \\
\hline
\end{tabular}

b. Análisis del efecto de la dosis de encalamiento dentro de la variedad

\begin{tabular}{|c|c|c|}
\hline \multirow{2}{*}{$\begin{array}{c}\text { Dosis de cal } \\
\left(\mathrm{t} \mathrm{ha}^{-1}\right)\end{array}$} & \multicolumn{2}{|c|}{ Variedad } \\
\cline { 2 - 3 } & Cayena lisa & Criolla \\
\hline 0 & $4,12 \mathrm{ab}$ & $3,82 \mathrm{a}$ \\
\hline 3 & $4,12 \mathrm{a}$ & $3,97 \mathrm{~b}$ \\
\hline 6 & $4,08 \mathrm{ab}$ & $3,97 \mathrm{~b}$ \\
\hline 9 & $4,06 \mathrm{~b}$ & $3,92 \mathrm{~b}$ \\
\hline
\end{tabular}

Promedios con letras distintas en la misma columna indican diferencia significativa según el test protegido de DMS $(P \leq 0,05)$.

de la región, podría presentar mecanismos de tolerancia al $\mathrm{Al}^{3+}$ más eficientes que los de Cayena lisa, explicados probablemente por los cambios en los ácidos orgánicos de los exudados de la raíz y a la proteína soluble de los ápices de esta, tal como lo proponen Le Van y Masuda (2004).

En los sistemas de producción de pequeños productores como los agricultores de piña en Córdoba, no es raro observar que al escoger la semilla de su próxima siembra, seleccionen material vegetal de manera natural a partir de plantas sanas y vigorosas, lo que indirectamente se traduce en una selección de ecotipos adaptados a las condiciones de los SSA típicos de la región; esto pudiera ser una explicación adicional al comportamiento más estable de 'Criolla' respecto a 'Cayena lisa'. 


\section{CONCLUSIONES}

Los resultados de la investigación permiten concluir que el encalamiento de los SSA de Ciénaga de Oro en el departamento de Córdoba (Colombia) no tuvo un efecto importante sobre la resistencia y el diámetro del fruto de la piña en las variedades Cayena lisa y Criolla. Sin embargo, el pH del fruto mostró un comportamiento diferencial dentro de las variedades al cambiar el nivel de encalamiento. En este sentido, la variedad Criolla presentó una respuesta más directa al encalamiento probablemente como consecuencia de su adaptación a los SSA de la región.

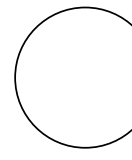

\section{REFERENCIAS BIBLIOGRÁFICAS}

Arias, C. y J. Toledo. 2000. Manual de manejo postcosecha de frutas tropicales. Papaya, piña, plátano, cítricos. En: http://www.fao.org/inpho/content/documents/vlibrary/ac304s/ac304s00.htm; consulta: junio de 2007.

Bartholomew, D. y S. Kadzimin. 1997. Pineapple. pp. 113156. En: Alvim, P. de T. y T.T. Kozlowski (eds.). Ecophysiology of tropical crops. Academic Press, NY.

Bartholomew, D.P. y E. Malézieux. 1994. Pineapple. pp. 243-291. En: Schaffer, B. y P.C. Anderson (eds.). Handbook on environmental physiology of fruit crops, Vol. II. Subtropical and tropical crops. CRC Press, Boca Raton, Florida, FL.

Bello, F. y M. Gómez. 2001. Recuperación de suelos sulfatados ácidos en el área del distrito de riego del Alto Chicamocha, Tibasosa. Trabajo de grado. Facultad de Ciencias Agropecuarias, Universidad Pedagógica y Tecnológica de Colombia, Tunja.

Bernal, M. 2006. Madurez prematura en piña afecta la cosecha. En:ElUniverso.http://archivo.eluniverso.com/2006/01/ 21/0001/71/953BE7C7FD69406B80382F2320F6F5FD. aspx; consulta: enero de 2006.

Carvalho, S.; C. Neves; R. Bürkle y C.J. Marur. 2005. Floral induction period and thermal time requirements from the flowering to the harvest period for Smooth Cayenne pineapple. Rev. Bras. Frutic. 27(3), 430-433.

Coppens, G. y F. Leal. 2003. Morphology, anatomy and taxonomy. pp. 13-32 En: Bartholomew, D.; R. Paull y K. Rohrbach (eds.). The pineapple: botany, production and uses. $\mathrm{CAB}$ International, $\mathrm{New}$ York, NY.
Cunha, G. y J. Cabral. 1999. Taxonomia, espécies, cultivares e morfologia. pp. 17-51. En: Cunha, G.; J. Cabral y L. Souza, L. (eds.). O abacaxizeiro: cultivo, agroindústria e economia. Embrapa Comunicação para Transferência de Tecnologia, Brasília.

De la Cruz, J.; P. Hernández; A. Rebolledo y H. García. 2007. Efecto de la aplicación de metil jasmonato sobre la fisiología poscosecha de piña (Ananas comosus cv. MD-2). pp. 82-91. En: Memorias V Congreso Iberoamericano de Tecnología Postcosecha y Agroexportaciones. Murcia, España.

Le Van, H. y T. Masuda. 2004. Physiological and biochemical studies on aluminum tolerance in pineapple. Aust. J. Soil Res. 42(5-6), 699-707.

Mathew, E.; R. Panda y M. Nair. 2001. Influence of subsurface drainage on crop production and soil quality in a low-lying acid sulphate soil. Agr. Water Manage. 47, 191-209.

New South Wales Department of Land and Water Conservation (NSWDLWC). 1999. Acid sulfate soil survey in NSW. Sidney.

Nowak, J. y A. Friend. 2005. Aluminum fractions in root tips of slash pine and loblolly pine families differing in $\mathrm{Al}$ resistance. Tree Physiol. 25, 245-250.

Paula, M.; H. Mesquita y F. Nogueira. 1998. Nutrição e Adubação do Abacaxizeiro. Informe Agropecuário, (Belo Horizonte) 19(195), 33-39.

Pérez F. y J. Martínez 2006. Aluminio. En: http://pdf.rincondelvago.com/aluminio_1.html; consulta: abril de 2006. 
Reinhardt, D. 2004. Quality gradients in 'Pérola' pineapple in function of fruit size and maturation stage. Rev. Bras. Frutic. 26(3), 544-546.

Salazar, R. 1994. Situación del cultivo de la piña en Colombia. pp. 151-159. En: Frutas tropicales. ICA, Bogotá.

Sierra, J.; C. Noel; L. Dufour; H. Ozier-Lafontaine; C. Welcker y L. Desfontaines. 2003. Mineral nutrition and growth of tropical maize as affected by soil acidity. Plant Soil 252, 215-226.

Taylor, G.; J. McDonald-Stephens; D. Hunter; P. Bertsch; D. Elmore; Z. Rengel y R. Reid. 2000. Direct measurement of aluminum uptake and distribution in single cells of Chara corallina. Plant Physiol. 123, 987-996.
Teixeira, L.; A. Spironello y P. Furlani. 2002. Split application of NPK fertilizers on pineapple. Rev. Bras. Frutic. 24(1), 219-224.

Universidad de Antioquia. 1993. Producción, transformación y comercialización. Pulpas de frutas tropicales. En: http://huitoto.udea.edu.co/FrutasTropicales/pina.html; consulta: junio de 2009.

Watanabe, T. y M. Osaki. 2002. Mechanisms of adaptation to high aluminum condition in native plant species growing in acid soils: a review. Comm. Soil Sci. Plant Anal. 33(7-8), 1247-1260.

Welch, S.; A. Christy; L. Isaacson y D. Kirste. 2009. Mineralogical control of rare earth elements in acid sulfate soils. Geochimica et Cosmochimica Acta 73, 44-64. 